\title{
BOUNDED APPROXIMATE UNITS AND BOUNDED APPROXIMATE IDENTITIES
}

\author{
JOSEF WICHMANN
}

\begin{abstract}
In this paper we establish the equivalence of the general concept of bounded approximate units in a normed algebra with the traditionally used notion of a bounded approximate identity. Furthermore, we investigate pointwise-bounded approximate units in commutative normed algebras.
\end{abstract}

Let $A$ be a normed algebra. A net $\left\{e_{\lambda}\right\}_{\lambda \in \Lambda}$ of elements in $A$ is called a bounded left approximate identity in $A$ if there exists a constant $K$ such that $\left\|e_{\lambda}\right\| \leqq K$ for all $\lambda \in \Lambda$ and $\lim _{\lambda \in \Lambda} e_{\lambda} x=x$ for all $x \in A$.

A normed algebra $A$ has bounded left approximate units if there exists a constant $K$ such that for every $x \in A$ and every $\varepsilon>0$ there exists an element $u \in A$ (depending on $x$ and $\varepsilon$ ) such that $\|u\| \leqq K$ and $\|x-u x\|<\varepsilon$.

We say that a normed algebra $A$ has pointwise-bounded left approximate units if for every $x \in A$ there exists a constant $K(x)$ such that for every $\varepsilon>0$ there exists an element $u \in A$ (depending on $x$ and $\varepsilon$ ) such that $\|u\| \leqq$ $K(x)$ and $\|x-u x\|<\varepsilon$.

Obviously, every normed algebra with a bounded left approximate identity has bounded left approximate units. Theorem 1 states the converse; the proof given below was kindly communicated to the author by Sadahiro Saeki and replaces our original proof. The argument is a modification of that given by $\mathrm{H}$. Reiter in $[\mathbf{1}$, p. 30$]$.

Furthermore, we show that a commutative normed algebra with pointwise-bounded approximate units has an approximate identity. The fact that we cannot assert the existence of a bounded approximate identity is illustrated by an example. But it turns out that the concept of pointwise-bounded approximate units is equivalent to the notion of a bounded approximate identity in commutative Banach algebras and also in commutative normed algebras which do not consist entirely of topological divisors of zero.

The general concept of approximate units in a normed algebra was

Received by the editors March 22, 1973.

AMS (MOS) subject classifications (1970). Primary 46H05, 46H25, 43A20.

Key words and phrases. Approximate unit, approximate identity, normed algebra, Banach algebra.

(c) American Mathematical Society 1973 
considered (under various names) by several authors; e.g. H. Reiter [1, pp. 27ff.] and H. C. Wang [2].

The author wishes to thank Professor R. S. Doran for constant encouragement and kind advice.

THEOREM 1. A normed algebra $A$ has left approximate units bounded by a constant $K$ if and only if $A$ has a left approximate identity bounded by the same constant $K$.

Proof. Let $A$ be a normed algebra with left approximate units bounded by a constant $K$. With the usual convention about the formal role of 1 , the assumption takes the following form: for every $x \in A$ and $\varepsilon>0$ there exists an element $u \in A$ such that $\|u\| \leqq K$ and $\|(1-u) x\|<\varepsilon$.

Now let $x_{1}, \cdots, x_{n}$ be any finite set of elements in $A$. Given $\varepsilon>0$, we can choose successively $u_{1}, \cdots, u_{n}$ in $A$ such that

$$
\left\|u_{i}\right\| \leqq K \text { and }\left\|\left(1-u_{i}\right) \cdot \cdots \cdot\left(1-u_{2}\right) \cdot\left(1-u_{1}\right) x_{i}\right\|<\varepsilon
$$

for all $i=1,2, \cdots, n$. Define $v$ in $A$ by $1-v=\left(1-u_{n}\right) \cdot \cdots \cdot\left(1-u_{2}\right) \cdot$ $\left(1-u_{1}\right)$. Then

$$
\begin{aligned}
\left\|x_{i}-v x_{i}\right\| & \\
& =\left\|\left[\left(1-u_{n}\right) \cdot \cdots \cdot\left(1-u_{i+1}\right)\right] \cdot\left[\left(1-u_{i}\right) \cdot \cdots \cdot\left(1-u_{1}\right) x_{i}\right]\right\| \\
& \leqq(1+K)^{n-i} \cdot\left\|\left(1-u_{i}\right) \cdot \cdots \cdot\left(1-u_{1}\right) x_{i}\right\|<(1+K)^{n} \cdot \varepsilon .
\end{aligned}
$$

Finally we choose $u$ in $A$ with $\|u\| \leqq K$ and $\|v-u v\|<\varepsilon$. Then for each $i=1,2, \cdots, n$ we have

$$
\begin{aligned}
\left\|x_{i}-u x_{i}\right\| & \leqq\left\|x_{i}-v x_{i}\right\|+\left\|(v-u v) x_{i}\right\|+\left\|u\left(x_{i}-v x_{i}\right)\right\| \\
& \leqq\left\|x_{i}-v x_{i}\right\|+\|v-u v\| \cdot\left\|x_{i}\right\|+K \cdot\left\|x_{i}-v x_{i}\right\| \\
& <(1+K)^{n} \cdot \varepsilon+\left\|x_{i}\right\| \cdot \varepsilon+(1+K)^{n+1} \cdot \varepsilon
\end{aligned}
$$

Hence, for every finite set $x_{1}, \cdots, x_{n}$ of elements in $A$ and every $\varepsilon>0$, there exists an element $u$ in $A$ such that $\|u\| \leqq K$ and $\left\|x_{i}-u x_{i}\right\|<\varepsilon$ for $i=1, \cdots, n$. Using a well-known construction $[1, \mathrm{p}$. 27] we conclude that the normed algebra $A$ has a left approximate identity bounded by the constant $K$.

THEOREM 2. A commutative normed algebra with pointwise-bounded approximate units has an approximate identity (possibly unbounded).

A commutative normed algebra $A$ which does not consist entirely of topological divisors of zero has pointwise-bounded approximate units if and only if $A$ has a bounded approximate identity.

Proof. Let $A$ be a commutative normed algebra with pointwisebounded approximate units. If $A$ does not consist entirely of topological 
divisors of zero, let $x_{0}$ be an element in $A$ which is not a topological divisor of zero; otherwise set $x_{0}=0$.

Now let $x_{1}, \cdots, x_{n}$ be any finite set of elements in $A$. Set

$$
K=K\left(x_{0}, x_{1}, \cdots, x_{n}\right)=\max \left\{K\left(x_{0}\right), K\left(x_{1}\right), \cdots, K\left(x_{n}\right)\right\} .
$$

Given $\varepsilon>0$ there exist elements $u_{0}, u_{1}, \cdots, u_{n}$ in $A$ such that $\left\|u_{i}\right\| \leqq K$ and $\left\|x_{i}-u_{i} x_{i}\right\|<\varepsilon$ for all $i=0,1, \cdots, n$.

Define $u$ in $A$ by $1-u=\left(1-u_{n}\right) \cdot \cdots \cdot\left(1-u_{1}\right)\left(1-u_{0}\right)$. Then

$$
\begin{aligned}
\left\|x_{i}-u x_{i}\right\| & =\left\|\left(1-u_{n}\right) \cdot \cdots \cdot\left(1-u_{1}\right)\left(1-u_{0}\right) x_{i}\right\| \\
& \leqq(1+K)^{n} \cdot\left\|\left(1-u_{i}\right) x_{i}\right\|<(1+K)^{n} \cdot \varepsilon .
\end{aligned}
$$

Hence, for every finite set $x_{1}, \cdots, x_{n}$ of elements in $A$ and every $\varepsilon>0$ there exists an element $u$ in $A$ such that $\left\|x_{i}-u x_{i}\right\|<\varepsilon$ for all $i=0,1, \cdots$, $n$. If $x_{0}$ is not a topological divisor of zero, it follows from the inequality $\left\|u x_{0}\right\|<\varepsilon+\left\|x_{0}\right\|$ that the elements $u$ are bounded by some fixed constant. Thus the assertion of Theorem 2 follows.

The next example shows that we cannot in general assert the existence of a bounded approximate identity.

EXAMPLE. Consider the commutative normed algebra

$$
A=\left\{\left(\lambda_{1}, \lambda_{2}, \cdots\right) \mid \lambda_{i} \text { complex and } \lambda_{i}=0 \text { for almost all } i\right\}
$$

with coordinatewise algebraic operations and norm defined by

$$
\left\|\left(\lambda_{1}, \lambda_{2}, \cdots\right)\right\|=\max _{i}\left|i \cdot \lambda_{i}\right| \text {. }
$$

Then $A$ has pointwise-bounded approximate units $u_{i}=(1, \cdots, 1,0,0, \cdots)$ Obviously, $A$ has no bounded approximate identity.

THEOREM 3. A commutative Banach algebra $A$ has pointwise-bounded approximate units if and only if $A$ has a bounded approximate identity.

Proof. Let $A$ be a commutative Banach algebra with pointwisebounded approximate units. Define $A_{n}=\left\{x \in A \mid \lim _{i} u_{i} x=x\right.$ for some sequence $\left(u_{i}\right)$ in $A$ with $\left.\left\|u_{i}\right\| \leqq n\right\}, n=1,2, \cdots$.

$A_{n}$ is a closed subset of $A$. For if $\left(x_{j}\right)$ is a sequence in $A_{n}$ with $\lim _{j} x_{j}=x$, then there exist sequences $\left(u_{i j}\right)_{i}$ in $A$ such that $\left\|u_{i j}\right\| \leqq n$ and $\lim _{i} u_{i j} x_{j}=x_{j}$. Then

$$
\begin{aligned}
\left\|x-u_{i j} x\right\| & \leqq\left\|x-x_{j}\right\|+\left\|x_{j}-u_{i j} x_{j}\right\|+\left\|u_{i j} x_{j}-u_{i j} x\right\| \\
& \leqq\left\|x-x_{j}\right\|+\left\|x_{j}-u_{i j} x_{j}\right\|+\left\|u_{i j}\right\| \cdot\left\|x_{j}-x\right\| \\
& \leqq(1+n) \cdot\left\|x-x_{j}\right\|+\left\|x_{j}-u_{i j} x_{j}\right\| ;
\end{aligned}
$$

choosing first $j$ and then $i$ large enough, it follows that $\left\|x-u_{i j} x\right\|$ can be made arbitrarily small. Hence $x \in A_{n}$. 
Since $A$ is the union of the sets $A_{n}, n=1,2, \cdots$, and $A$ is a Banach space, it follows from the Baire category theorem that some $A_{n}$ has nonempty interior. Thus $B\left(x_{0}, \delta\right)=\left\{x \in A \mid\left\|x-x_{0}\right\|<\delta\right\}$ is a subset of $A_{m}$ for some $x_{0} \in A, \delta>0$ and $m$. We will show that $B(0, \delta)=\{x \in A \mid\|x\|<$ $\delta\}$ is a subset of $A_{(2+m) m}$. Let $x \in B(0, \delta)$; then $x=\left(x+x_{0}\right)-x_{0}$ with $x+x_{0}$ and $x_{0}$ in $B\left(x_{0}, \delta\right)$. Hence there exist sequences $\left(u_{i}\right)$ and $\left(v_{i}\right)$ in $A$ such that $\left\|u_{i}\right\| \leqq m,\left\|v_{i}\right\| \leqq m, \lim _{i} u_{i}\left(x+x_{0}\right)=x+x_{0}$ and $\lim _{i} v_{i} x_{0}=x_{0}$. Set $w_{i}=u_{i}+v_{i}-u_{i} v_{i}$; then $\left(w_{i}\right)$ is a sequence in $A$ with $\left\|w_{i}\right\| \leqq(2+m) m$ and $\lim _{i} w_{i} x=x$; i.e. $x$ is in $A_{(2+m) m}$.

Since $\lambda \cdot A_{(2+m) m}$ is a subset of $A_{(2+m)_{m}}$ for any scalar $\lambda$, it follows that $A_{(2+m)_{m}}=A$; i.e. $A$ has bounded approximate units and so, by Theorem 1 , $A$ has a bounded approximate identity.

ADDED IN PROOF. It was stated by M. Altman (Contracteurs dans les algèbres de Banach, C.R. Acad. Sci. Paris Sér. A 274 (1972), A399-A400; Lemme 1) that every Banach algebra with bounded left approximate units has a bounded left approximate identity. The proof will appear in his paper on Contractors, approximate identities and factorization in Banach algebras in the Pacific J. Math.

It was proved by Teng-sun Liu, Arnoud van Rooij and Ju-kwei Wang (Projections and approximate identities for ideals in group algebras, Trans. Amer. Math. Soc. 175 (1973), 469-482; Lemma 12) that every commutative Banach algebra with pointwise-bounded approximate units has bounded approximate units.

\section{REFERENCES}

1. H. Reiter, $L^{1}$-algebras and Segal algebras, Springer-Verlag, Berlin and New York, 1971.

2. H. C. Wang, Nonfactorization in group algebras, Studia Math. 42 (1972), 231-241.

Department of Mathematics, Texas Christian University, Fort Worth, Texas 76129 